Journal of

Industrial Textiles

\title{
Development and blood compatibility evaluation of novel fibrous textile scaffold based on PU amalgamated with Alternanthera sessilis (AS) oil for bone tissue engineering
}

\begin{tabular}{|c|l|}
\hline Journal: & Journal of Industrial Textiles \\
\hline Manuscript ID & JIT-19-0010.R1 \\
\hline Keywords: & $\begin{array}{l}\text { incorporation < Chemistry, spinning < Fabrication, composites < } \\
\text { Materials, Fibrous materials }\end{array}$ \\
\hline Abstract: & $\begin{array}{l}\text { In the recent decade, the growth of medical textiles is enormous and it } \\
\text { paves the sustained development in the quality of life. In this research, } \\
\text { an electrospun textile scaffold comprising of polyurethane (PU) } \\
\text { impregnated with Alternanthera sessilis (AS) oil was developed for the } \\
\text { bone tissue engineering. Morphology analysis showed that the addition } \\
\text { of AS oil in the PU membrane resulted in reduced fiber diameter (821 } \pm \\
\text { 140.87 nm) compared to the pristine PU (890 } \pm 119.11 \text { nm). The } \\
\text { presence of AS oil in PU membrane was confirmed through hydrogen } \\
\text { bond formation as revealed in the infrared (IR) analysis. Further, the PU } \\
\text { blended with AS oil showed hydrophobic (113.70 } \pm \text { P.215) and improved } \\
\text { surface roughness (329 nm) than the pristine PU (Contact angle - 100 } \\
\pm 0.5774 \text { and Ra - 313 nm). Moreover, the blood compatibility } \\
\text { assessments revealed that the developed biocomponent PU/AS oil } \\
\text { membrane possess enhanced anticoagulant nature compared to the } \\
\text { pristine PU. Finally, the newly developed biocomponent PU/AS oil } \\
\text { membrane with reduced fiber diameter, hydrophobic behavior, improved } \\
\text { surface roughness and enhanced blood compatibility enabled them as a } \\
\text { potential candidate for bone tissue regeneration. }\end{array}$ \\
\hline
\end{tabular}

\section{SCHOLARONE Manuscripts}




\title{
Development and blood compatibility evaluation of novel fibrous textile scaffold based on PU amalgamated with Alternanthera sessilis (AS) oil for bone tissue engineering
}

\begin{abstract}
In the recent decade, the growth of medical textiles is enormous and it paves the sustained development in the quality of life. In this research, an electrospun textile scaffold comprising of polyurethane (PU) impregnated with Alternanthera sessilis (AS) oil was developed for the bone tissue engineering. Morphology analysis showed that the addition of AS oil in the PU membrane resulted in reduced fiber diameter $(821 \pm 140.87 \mathrm{~nm})$ compared to the pristine PU $(890 \pm 119.11$ $\mathrm{nm})$. The presence of AS oil in PU membrane was confirmed through hydrogen bond formation as revealed in the infrared (IR) analysis. Further, the PU blended with AS oil showed hydrophobic $\left(113.7^{\circ} \pm 3.215\right)$ and improved surface roughness $(329 \mathrm{~nm})$ than the pristine PU (Contact angle $100^{\circ} \pm 0.5774$ and $\mathrm{R}_{\mathrm{a}}-313 \mathrm{~nm}$ ). Moreover, the blood compatibility assessments revealed that the developed biocomponent PU/AS oil membrane possess enhanced anticoagulant nature compared to the pristine PU. Finally, the newly developed biocomponent PU/AS oil membrane with reduced fiber diameter, hydrophobic behavior, improved surface roughness and enhanced blood compatibility enabled them as a potential candidate for bone tissue regeneration.
\end{abstract}

Keywords: Polyurethane, Alternanthera sessilis, Fibrous textile scaffold, Bone tissue engineering. 


\section{Introduction}

The standard methods utilized for bone repair are autologous and allogeneic transplantations. However, it is limited due to certain drawbacks like limited donor site supply, immune rejection, donor site morbidity and transfer of pathogens [1]. With the development of technology, the growth of bone tissue engineering was found to be enormous and demanding for over the years mainly due to their some advantages like limitless supply and no disease transmission. The objective of bone tissue engineering is to provide functional bone regeneration through a combination of scaffolds, cells and growth factors [1]. Among these, the scaffold plays a vital role in mimicking the native extra cellular matrix (ECM) of human tissue. Further, they also help in supporting the cell adhesion and proliferation for new tissue growth. Recently, the fabric based on textile technologies were widely utilized in different application areas such as liquid fuel purification, textile based sensors, protective clothing and tissue engineering applications [2-7]. It is possible to fabricate complex structures and three dimensional designs with the help of textile technologies. Further, the studies have shown that functionalized fabrics were reported to possess outstanding biological properties with less toxicity [8-13] Although several techniques like weaving, knitting, braiding were utilized in the fabrication of fabrics, usage of electrospinning to produce non-woven scaffolds is indispensable [14].

Electrospinning is a versatile and cost effective technique which could able to fabricate fibres in ranging from micrometre to nanometre. The nanofibers fabricated through electrospinning technique was widely utilized in biomedical applications owing to its high surface area to volume ratio, microporous structure and also ability to mimic the extracellular matrix (ECM) structure of the human tissue [15]. Further, the nanofibers enhance cell adhesion, proliferation and growth for remodelling of the damaged tissue. Further, the important properties considered in the design of scaffolds for bone tissue engineering were biodegradable, biocompatible, porosity, non-toxic and good mechanical properties $[16,17]$. A wide range of synthetic and natural polymers have been employed in obtaining nanofibers for the past few years either in pure or blended form. PU is one among these which possesses the good mechanical property and water insolubility which finds applications in biosensors, protective cloths and enhancing epithelial growth [18]. It was reported to be biocompatible, biodegradable, good oxidation and thermal stability $[19,20]$. Further, it can be inferred that PU based scaffolds hold great potential in tissue engineering applications like skin, bone and cardiac [21-24]. In this study, Tecoflex EG-80A polyurethane was used to fabricate the bone scaffold.

Hydroxyapatite nanoparticles (nano HA), a prospective biomaterial which is utilized for making bone substitutes owing to their biocompatible, biodegradable nature, osteo-conductive, osteoinductive and osteo-integrative properties [25]. Apart from these outstanding properties, it was

found to be risky when they were applied in the clinical applications due to their nano scale particle 
size [26]. Despite their promising potential in the clinical applications, the safety and toxicity of HA are still considered as a challenging one. Hence, it forces the researchers to find alternatives to the hydroxyapatite particles. In Asian countries, the essential oil plays an important role in alleviating the pains created at the bone joints. The essential oils derived from the plants was reported to be less toxic and cost effective. Recent research suggested the use of oils in bone tissue engineering applications [6,7]. This motivated us to use Alternanthera sessili (AS) oil in this research.

AS is a member of Amaranthaceae family which will grow both in wetlands and uplands. It is rich in vitamin A, dietary fibre and also contains various bioactive constituents like flavonols, steroids, tannins, triterpenoids, $\beta$ '-sitosterol, campesterol, stigmasterol, and lupeol. The chemical structure of AS is indicated in Figure 1. Further, it also possesses minerals like sodium (Na), potassium (K), magnesium $(\mathrm{Mg})$, calcium (Ca) and a small amount of zinc, copper and iron ( $\mathrm{Zn}, \mathrm{Cu}$ and $\mathrm{Fe}$ ) [27]. AS is reported as a remedy for various ailments like night blindness, dysentery, intestinal cramps, anaemia, hepatitis, diarrhoea, bronchitis, tight chest, lung troubles, asthma, treating fever and wounds [28-30]. Further, it has been reported that this plant possesses various medicinal activities like antibacterial, antiulcer, antipyretic, diuretic and hematinic [28]. Several researches indicated its versatile nature in biomedical applications. However, there is no single report on the use of AS oil in tissue engineering application. The aim of the study is to fabricate an electrospun novel bone scaffold based on PU incorporated with the AS oil. In addition, the fabricated membranes was subjected to the physico-chemical characterization, blood compatibility assessments and cytocompatibility studies. 


\section{Materials and Methodology}

\section{Materials}

Medical grade Tecoflex EG-80A polyurethane (PU) was obtained from LubriZol, USA and N, $\mathrm{N}$-dimethylformamide (DMF) was supplied by Merck Millipore, Germany. AS oil was obtained from Thurgas Trading, Pulau Pinang, Malaysia. Phosphate buffered saline (PBS, biotech Grade) and sodium chloride physiological saline $(0.9 \% \mathrm{w} / \mathrm{v})$ used in the blood compatibility assays were supplied by Sigma-Aldrich, Malaysia. The Activated partial thromboplastin time (APTT) and Prothrombin time (PT) reagents such as rabbit brain activated cephaloplastin, calcium chloride $(0.025 \mathrm{M})$, and thromboplastin (Factor III) were purchased from Diagnostic Enterprises, India. 


\section{Preparation of PU and biocomponent PU/AS electrospun solutions}

To prepare 9 wt \% of homogeneous PU solution, $480 \mathrm{mg}$ of PU was dissolved in $4.5 \mathrm{ml}$ of DMF and stirred overnight. Similarly, $4 \mathrm{v} / \mathrm{v} \%$ of homogeneous AS solution was made by adding $120 \mu 1$ of AS in $3 \mathrm{ml}$ of DMF respectively and stirred for $1 \mathrm{hr}$ maximum. Finally, Polyurethane/AS solution with the ratio of $8: 1 \mathrm{v} / \mathrm{v} \%$ was obtained by slowly adding the prepared homogeneous PU and AS solution and stirred for $2 \mathrm{hr}$ to obtain uniform dissolution.

Fabrication of electrospun PU and biocomponent PU/AS oil membranes for bone scaffolds

The electrospun membranes were fabricated through electrospinning technique (Progene Link Sdn Bhd, Malaysia) operated in room temperature with humidity of 55\%. To begin, the prepared homogeneous solution was loaded in $10 \mathrm{ml}$ syringe attached with $18 \mathrm{G}$ stainless steel needle (outer diameter of $1.28 \mathrm{~mm}$ and an inner diameter of $0.92 \mathrm{~mm}$, Unicontrols Singapore Pte. Ltd) and fitted to the syringe pump. In our study, both PU and biocomponent PU/AS oil membranes were electrospun at a flow rate of $0.5 \mathrm{ml} / \mathrm{hr}$ with an applied voltage of $10 \mathrm{kV}$. The deposited fibers membranes were deposited on the static collector drum which was placed $20 \mathrm{~cm}$ from the needle. Finally, the obtained fibers were dried under vacuum at room temperature for $24 \mathrm{~h}$ to remove any residual DMF. The images of electrospun PU and biocomponent PU/AS oil membranes were shown in Figure 2.

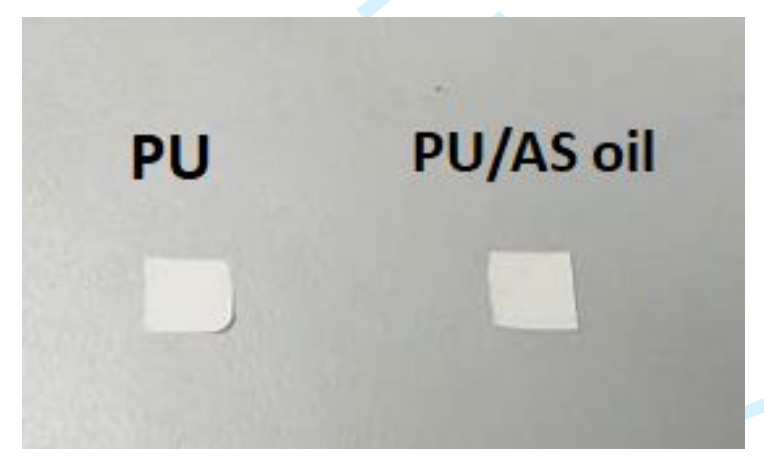

Figure 2 Electrospun membranes of PU and biocomponent PU/AS oil membrane 


\section{Scanning electron microscopy (SEM)}

SEM (Hitachi Tabletop TM3000, Tokyo, Japan) was utilized to analyze the fibrous morphology and their fiber diameters. Before attaining photographs, the samples were gold coated with gold at $20 \mathrm{~mA}$ for $2 \mathrm{~min}$ and the scanning was performed. The photographs were captured at different magnifications at an accelerated voltage of $10 \mathrm{kV}$. Using Image $\mathbf{J}$ software, the average fiber diameter and fiber distribution were calculated by measuring at least individual 50 fibers randomly from the captured image with a magnification of $\times 2.5 \mathrm{k}$.

\section{Contact angle measurements}

The contact angles for the electrospun membranes were measured through a video contact angle (VCA) measurement unit (AST Products, Inc., Billerica, MA, USA). The contact angle of the electrospun membranes was measured in static mode. A droplet of deionized water with a size of $0.5 \mu 1$ was placed on the fabricated membranes and the image of the water droplet was captured using video cam. Using computer integrated software, the contact angle was calculated and the experiment was repeated for three different trials to measure the average value.

\section{Atomic force microscopy (AFM)}

Surface analysis was carried out in an AFM equipment (NanoWizard $\AA$, JPK Instruments, Berlin, Germany) under a normal atmosphere to measure the average surface roughness of the electrospun membranes. The surface roughness of the electrospun membranes was determined by scanning in $20 \times 20 \mu \mathrm{m}$ area with $256 \times 256$ pixels.

\section{Chemical and structural characteristics}

Infrared (IR) spectrum of the electrospun membranes was inspected in Fourier transform infrared spectroscopy (FTIR) unit (Nicolet IS 5, Thermo Fischer Scientific, Waltham, MA, USA) equipped with zinc selenium $(\mathrm{ZnSe})$ as an attenuated total reflectance (ATR) crystal. The absorption bands of the electrospun membranes were measured once at a wavelength of 600 and $4000 \mathrm{~cm}^{-1}$ with a resolution of $4 \mathrm{~cm}^{-1}$.

\section{TGA analysis}

TGA equipment (PerkinElmer, Waltham, MA, USA) was utilized to determine the thermal behaviour of the electrospun membranes. A small piece weighing $3 \mathrm{mg}$ is placed in an aluminium pan and the heating was performed once under a nitrogen atmosphere at a rate of $10{ }^{\circ} \mathrm{C} \min ^{-1}$ in the temperature range of $30^{\circ} \mathrm{C}$ to $1000^{\circ} \mathrm{C}$. 


\section{Coagulation assay}

\section{Prothrombin Test}

Measurement of prothrombin time was used to predict the extrinsic pathway. To start the assay, $50 \mu 1$ of platelet poor plasma (PPP) was applied on the surface of the electrospun membranes and incubated at $37^{\circ} \mathrm{C}$. In next step, it was added with $50 \mu \mathrm{l}$ of $\mathrm{NaCl}$-thromboplastin (Factor III) containing $\mathrm{Ca}^{2+}$ ions. Finally, the time taken for the blood clot was measured using the stopwatch and a steel hook [6,7].

\section{Activated Partial Thromoplastin Test (APTT)}

On the other hand, the measurement of APTT was used to predict the intrinsic pathway. To start the assay, the samples were incubated with $50 \mu \mathrm{l}$ of PPP at $37^{\circ} \mathrm{C}$. After, it was further incubated with $50 \mu \mathrm{l}$ rabbit brain encephalin for $5 \mathrm{~min}$ followed by adding calcium chloride $(0.025 \mathrm{M})$. Finally, the time taken for the blood clot was measured using the stopwatch and a steel hook [6,7].

\section{Hemolysis Test}

To begin the assay, the samples were soaked with physiologic saline at $37^{\circ} \mathrm{C}$ for $30 \mathrm{~min}$. Initially, the citrated blood was diluted in saline at a ratio of $4: 5 \mathrm{v} / \mathrm{v}$ and then the samples were incubated for $60 \mathrm{~min}$ at $37^{\circ} \mathrm{C}$. Then, these mixtures were centrifuged at $3000 \mathrm{rpm}$ for $5 \mathrm{~min}$. Finally, the clear supernatant was pipetted and the absorbance was measured at $542 \mathrm{~nm}$ which express the release of hemoglobin. The percentage of hemolysis was calculated as per the formula as discussed earlier [6,7].

\section{Statistical Analysis}

All experiments were performed three times individually unless stated otherwise. The statistical significance was determined through Unpaired t-test. The obtained results from all experiments are conveyed as mean $\pm \mathrm{SD}$. A representative of three images is shown for qualitative experiments.

\section{Result and Discussion}

Figure $3 \mathrm{a}$ and $3 \mathrm{~b}$ illustrate the morphology of PU and biocomponent PU/AS oil membrane determined through SEM analysis. It was observed from the SEM images that the electrospun PU and biocomponent PU/AS oil membranes showed continuous fibers without any beads. The calculated fiber diameters of PU and biocomponent PU/AS oil membrane using Image J were found to be $0.890 \pm 0.119 \mu \mathrm{m}$ and $0.821 \pm 0.141 \mu \mathrm{m}$ respectively. The corresponding fiber diameter distribution curve was indicated in Figure $3 \mathrm{c}$ and $3 \mathrm{~d}$. The pristine showed 6 fibers in the rage of 700-800, 15 fiber in the range of 800-900, 25 fibers in the range of 900-1000 and 4 fibers in the range of 1000-1100 respectively. The PU/AS oil showed 20 fibers in the rage of 700-800, 19 fiber in the range of 800-900, 7 fibers in the range of 900-1000 and 4 fibers in the range of 
1000-1100 respectively. The fiber diameter measurements revealed the reduction in the fiber diameter of PU membrane when incorporating of AS oil and this might be due to the decrease in the polymer concentration while adding AS oil into the polyurethane membrane. Further, this may be due to the plasticizing effect of the AS oil with the PU molecules causing a reduction in the glass transition temperature. This may be corroborated using our SEM results as we observed smooth morphology for biocomponent PU/AS membrane compared to the more rigid pristine PU. Jaganathan et al developed polyurethane scaffold for bone tissue engineering added with corn and neem oil. It was reported that the addition of corn and neem oil resulted in the reduction of the fiber diameter of the pristine PU which resembles our findings. They concluded that fabricated composite as a suitable candidate for bone tissue engineering [6]. Our electrospun biocomponent PU/AS oil membrane exhibited reduced fiber diameter which might be conducive to the bone tissue engineering.

(a)

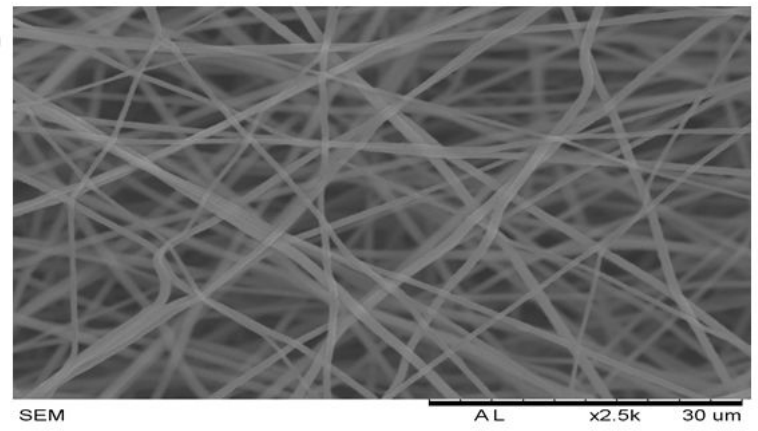

(c)

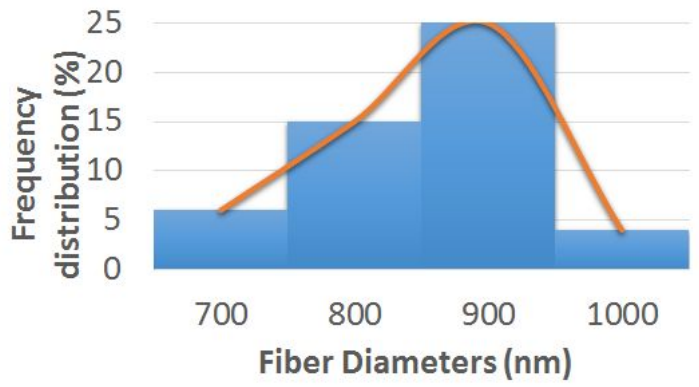

(b)

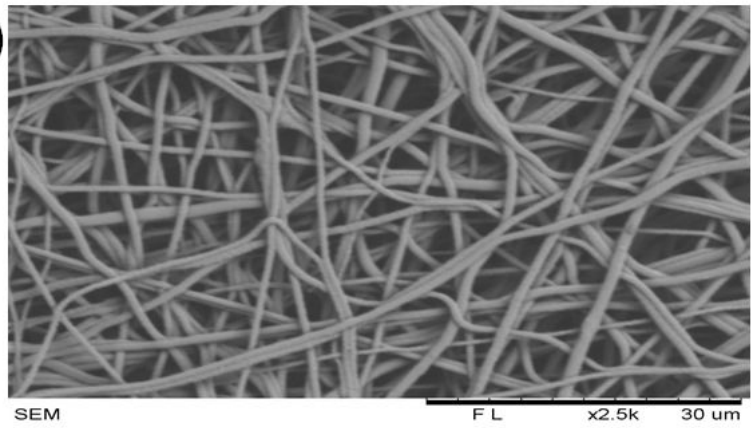

(d)

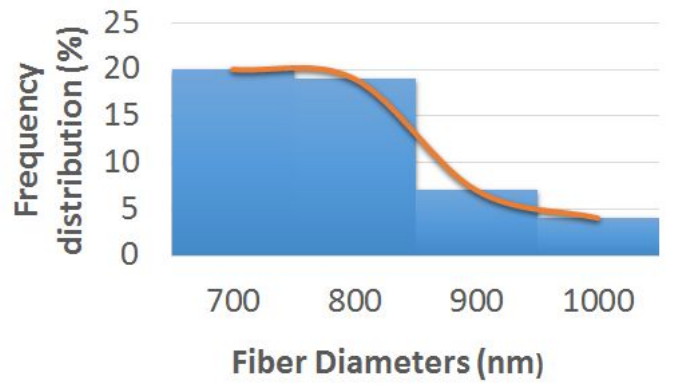

Figure 3 SEM images of a) PU membrane b) biocomponent PU/AS membrane and Fiber diameter distribution of c) PU membrane d) biocomponent PU/AS membrane

Figure $4 \mathrm{a}$ and $4 \mathrm{~b}$ present the IR spectrum of developed PU and biocomponent PU/AS oil membrane. The spectra of PU shows a wide band at $3323 \mathrm{~cm}^{-1}$ indicates the N-H stretching vibrations and the peak at $1597 \mathrm{~cm}^{-1}$ and $1531 \mathrm{~cm}^{-1}$ indicates the $\mathrm{N}-\mathrm{H}$ bending vibrations. The symmetric and asymmetric $\mathrm{CH}_{2}$ stretching vibrations were observed at the peak $2939 \mathrm{~cm}^{-1}$ and $2853 \mathrm{~cm}^{-1}$, while the peak at $1413 \mathrm{~cm}^{-1}$ indicates the $\mathrm{CH}_{2}$ bending vibrations respectively. The sharp peaks at $1730 \mathrm{~cm}^{-1}$ and $1703 \mathrm{~cm}^{-1}$ represents the $\mathrm{C}=\mathrm{O}$ stretching vibration and further peaks 
seen between 1200-1000 $\mathrm{cm}^{-1}$ represents the stretching vibration of C-O-C and C-OH respectively [31]. From the spectra of biocomponent PU/AS oil membrane, it was noted no new band was formed but the intensity of PU peak was increased with the addition of AS oil due to strong hydrogen bond formation as denoted in Figure $4 \mathrm{~b}$ [21]. Further, there was a peak shift found which also depicts the interaction of PU with AS oil. The CH band in PU from $2939 \mathrm{~cm}^{-1}$ was shifted to $2924 \mathrm{~cm}^{-1}$ in biocomponent PU/AS oil membrane which confirms the presence of AS oil in the PU [32]. Similar findings of peak shift and change in peak intensity were reported in oils incorporated polyurethane scaffold $[6,21]$. The formation of hydrogen bonds and peak shift was due to the interaction of $\mathrm{OH}$ and $\mathrm{CH}$ molecules in the $\mathrm{AS}$ oil with molecules of the polyurethane.

(a)

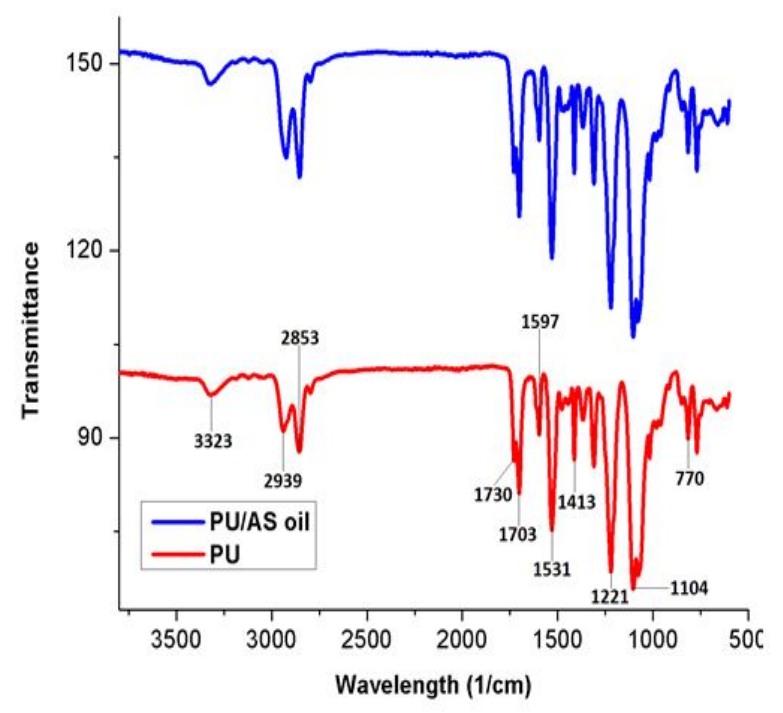

(b)

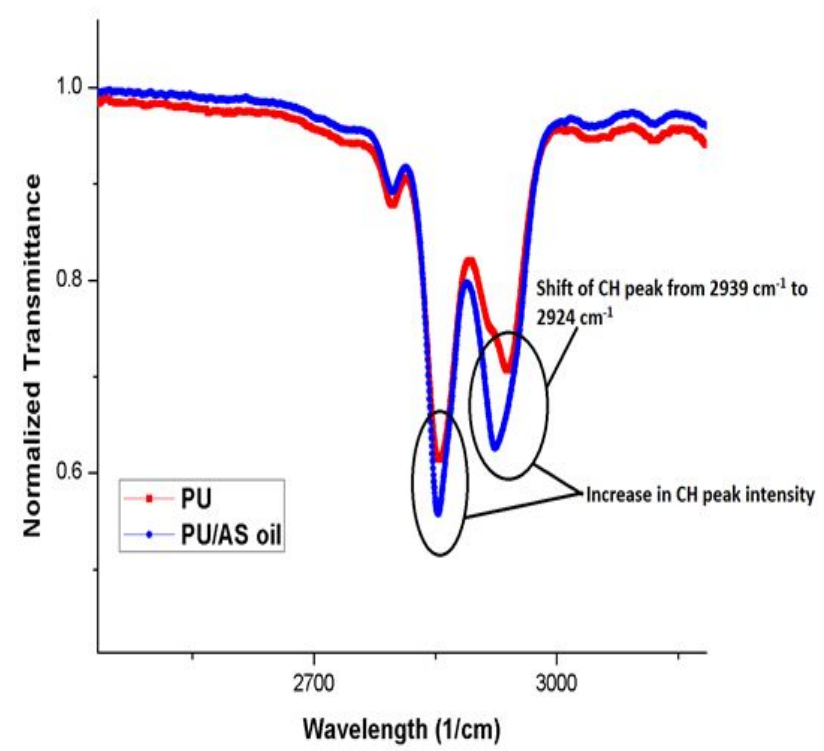

Figure 4 IR spectrum of PU membrane and biocomponent PU/AS membrane

The contact angle measurements of electrospun PU and biocomponent PU/AS oil membrane were discussed. It was observed that the wettability of biocomponent PU/AS membrane was lower than PU membrane. The contact angle of the pristine PU membrane was found to be $100^{\circ} \pm 0.5774$, while the contact angles of the biocomponent PU/AS oil membrane were found to $113.7^{\circ} \pm 3.215$ respectively. Hence, the incorporation of AS oil increased the hydrophobic nature of the 
polyurethane. Cui et al. developed poly(D,L-lactide) and poly(D,L-lactide)-poly(ethylene glycol) membranes using electrospinning. It was observed that the electrospun scaffold with smaller fiber diameter resulted in the improvement of contact angle [33]. In another research, Ceylan et al. fabricated electrospun scaffold utilizing polystyrene and polyvinyl chloride fibers. It was shown that the water contact angle was enhanced when the diameter of fiber becomes smaller [34]. In our study, the developed biocomponent PU/AS oil membrane showed a smaller fiber diameter than PU membrane which might have favored the increase in the contact angle. Cui et al reported that the decrease in fiber diameters and the pore sizes would cause higher air entrapment in the surface which might favour the larger contact angle [33]. Our smaller fiber diameter of the fabricated biocomponent PU/AS oil membrane might cause higher air entrapment which favoured the larger contact angle. In a recent study, Jaganathan et al fabricated polyurethane scaffold added with sunflower and neem oil for bone tissue engineering. The contact angle of the fabricated biocomponent PU/sunflower oil was reported to be $112^{\circ} \pm 1$ and exhibited enhanced cellular response than the pristine PU [7]. Hence, our biocomponent PU/AS oil membrane showed contact angle within the reported range and might be suitable for the bone tissue engineering.

(a)

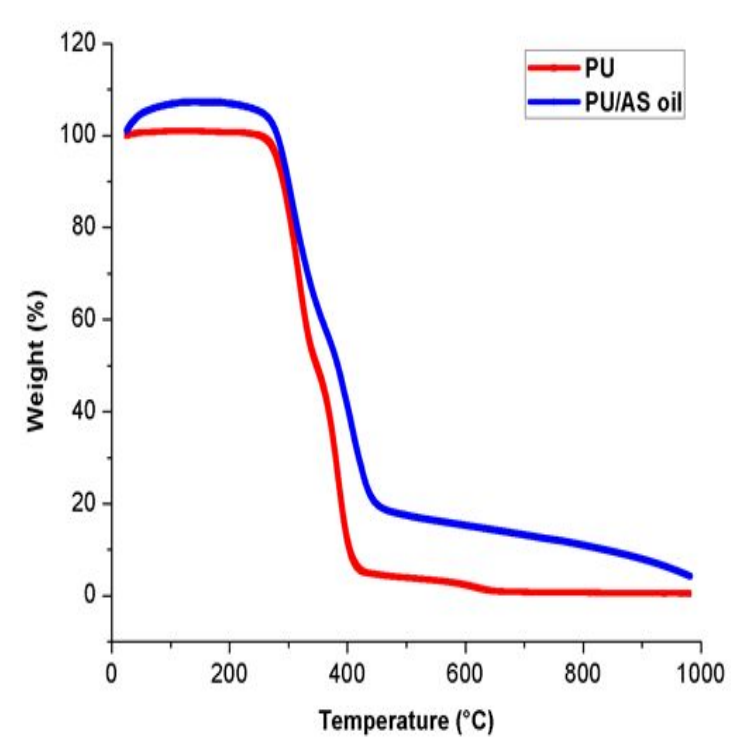

(b)

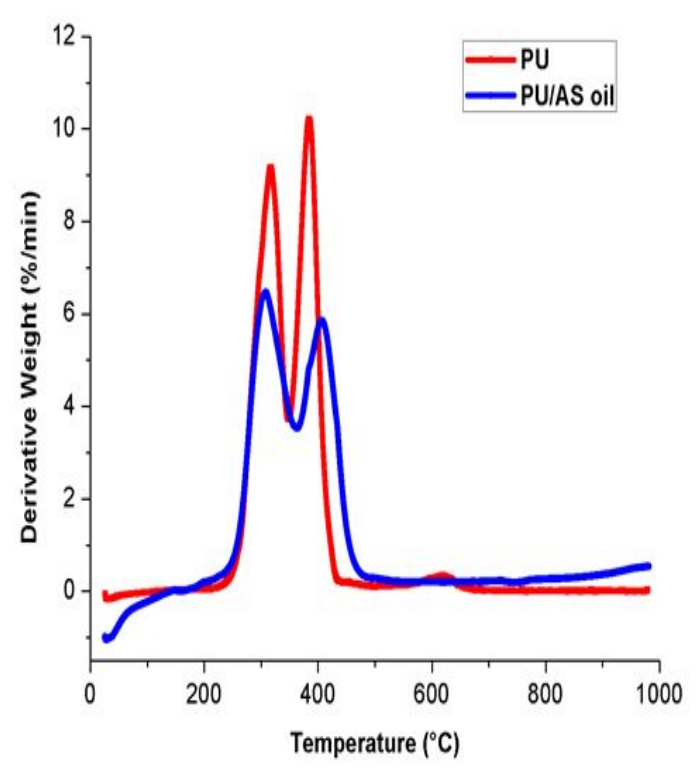

Figure 5 a) TGA and b) DTG analysis of PU membrane and biocomponent PU/AS membrane 
Figure 5a and 5b indicates the TGA and DTG curves of the electrospun PU and biocomponent PU/AS oil membrane. The electrospun PU membrane showed no change in thermal behaviour with the addition of AS oil. It was noted from the TGA that both PU and biocomponent PU/AS oil membrane starts to degrade initially at $276^{\circ} \mathrm{C}$ respectively. Further, from the figure it was evident that biocomponent PU/AS membrane showed initial weight gain which might be due to the bonding of AS oil molecules with PU. This observation is further correlated with the FTIR results which depict the formation of hydrogen bonding as confirmed by the shifting of $\mathrm{CH}$ band in PU from $2939 \mathrm{~cm}^{-1}$ to $2924 \mathrm{~cm}^{-1}$ in biocomponent PU/AS oil membrane. Moreover, at $1000^{\circ} \mathrm{C}$, the remaining weight residue percentage of pure PU membrane was observed to be $0.47 \%$, while for the electrospun biocomponent PU/AS oil membrane, it was increased to $1.58 \%$ which might due to higher unburnable/ash content. Further, the derivate weight loss curve depicted that the weight loss in the electrospun biocomponent PU/AS oil membrane was lower than the pure PU. From the DTG diagram, it was revealed the electrospun PU membrane showed three weight loss in which the first loss occurs at $223^{\circ} \mathrm{C}$ to $348^{\circ} \mathrm{C}$ (degradation of urethane groups), the second loss begins $348^{\circ} \mathrm{C}$ to $446^{\circ} \mathrm{C}$ (degradation of ester bonds) and the final loss was seen at $557^{\circ} \mathrm{C}$ to $684^{\circ} \mathrm{C}$ (degradation of aromatic) respectively $[35,36]$. In the case of electrospun biocomponent PU/AS membrane, it showed two weight loss peaks in which the first weight loss occurs at $225^{\circ} \mathrm{C}$ to $363^{\circ} \mathrm{C}$ and the second loss at $363^{\circ} \mathrm{C}$ to $514^{\circ} \mathrm{C}$ respectively. The first loss of biocomponent PU/AS oil may be attributed to the degradation of the urethane group. The second loss seems to be wider compared to the PU and this may be due to the melting of ester and partly aromatic groups. It was observed from the curve that the weight loss peak intensity of the fabricated biocomponent PU/AS membrane was observed to lower than the pristine PU indicating their reduced weight loss.

Figure 6 indicates the AFM images of PU and biocomponent PU/AS oil membrane which depicts the average surface roughness. It was noted that the incorporation of AS into the PU membrane increased the average surface roughness. The calculated surface roughness for the pristine PU membrane was found to be $313 \mathrm{~nm}$, while the electrospun biocomponent PU/AS oil membrane showed $\mathrm{Ra}$ of $329 \mathrm{~nm}$ respectively. The increase in the surface roughness was due to the addition of AS oil into the pristine PU. The bioactive constituents present in the AS oil might have favoured the increase in the surface roughness value. A similar increase in surface roughness was observed in a recent study by Jaganathan et al., 2017. In their work, electrospun PU scaffold added mustard oil was developed. They showed an increase in the surface roughness than the pristine PU due to the bioactive constituents present in the mustard oil [37]. It has been reported that the measurement of surface roughness was correlated to the adhesion, proliferation and growth of osteoblastic cell. Rough surfaces enhance the osteoconductive process by favouring entrapment of fibrin protein and adhesion of an osteogenic cells [38]. Our fabricated biocomponent PU/AS oil membrane showed rougher surfaces which might be suitable for enhancing the osteoconductive process. 
(a)

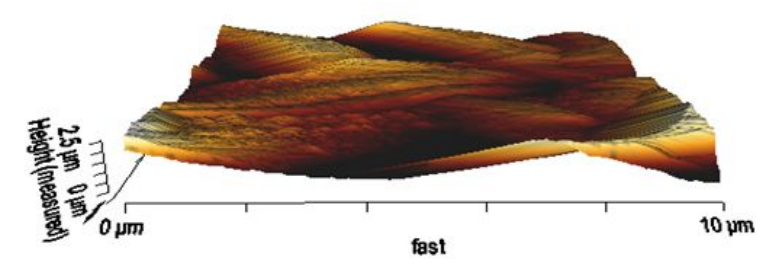

(b)

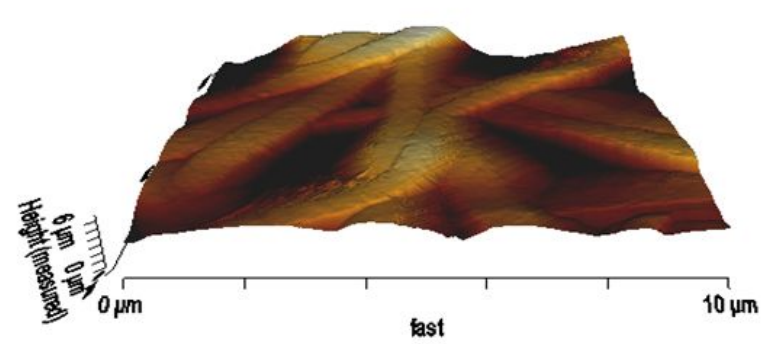

Figure 6 AFM images of a) PU membrane b) biocomponent PU/AS membrane

The blood compatibility assessments of the electrospun PU and biocomponent PU/AS oil membrane measured through APTT and PT assay. Both APTT and PT assessments revealed marginally enhanced blood clotting time exhibited by the biocomponent PU/AS oil membrane than the PU membrane. In APTT assay, the blood clotting time for PU membrane was found to be $175.3 \pm 4.041 \mathrm{~s}$ while the electrospun biocomponent PU/AS oil membrane showed blood clotting time of $183 \pm 2.646 \mathrm{~s}$ respectively. Similarly, in PT assay, the blood clotting time for PU membrane was found to be $76.33 \pm 2.517 \mathrm{~s}$ while the electrospun biocomponent PU/AS oil membrane showed blood clotting time of $80.67 \pm 1.528 \mathrm{~s}$ respectively. 
The anticoagulant nature of the biocomponent PU/AS oil is higher than the pure polyurethane but the results are not statistically significant. However, it can be inferred that the introduction of AS oil does not have any negative influence on anticoagulant behavior of the fabricated membrane. Further, the release of haemoglobin was measured for all electrospun membranes through hemolysis assay and the obtained results were presented. The assay results showed that the hemolytic percentage for the electrospun biocomponent PU/AS oil membrane were lowered than the pristine PU. The measured hemolytic index for electrospun biocomponent PU/AS oil membrane was found to be $1.35 \%$, while the pristine PU membrane induced hemolytic index of $2.48 \%$. It was reported when the measured hemolytic index was below $2 \%$, then the material is called as non-hemolytic material. Hence, the developed biocomponent PU/AS oil membrane showed hemolytic index below than $2 \%$ and it was a non-hemolytic material [6,7]. In this experiment, the percentage of hemolysis was significantly lower than the polyurethane. This shows our fabricated biocomponent PU/AS oil membrane have relatively edge over the pure polyurethane. Reduced hemolysis with the anticoagulant nature may collectively promote the fabricated biocomponent PU/AS oil membrane as a candidate with enhanced blood compatibility for bone tissue engineering applications. Jaganathan et al fabricated polyurethane scaffold added with corn and neem oil. The reported blood clotting time of the PU/corn/neem oil was found to increase compared to the pristine PU. APTT and PT of PU/corn/neem oil were found to be $3.47 \%$ and $3.75 \%$ enhancement than the control suggesting a suitable candidate for bone tissue engineering [6]. In our study, APTT and PT time of PU/AS oil found to the $4.39 \%$ and 5.68\% enhancement than the pristine PU. Hence, the developed biocomponent PU/AS oil membrane showed better compatibility than the reported values suggesting its suitability for bone tissue engineering. It was reported that the blood compatibility was influenced by surface parameters such as smaller fiber diameter [39], small surface tension [40] and enhanced surface roughness [37]. In our study, the fabricated biocomponent PU/AS oil membrane showed smaller fiber diameter and enhanced surface roughness which might have influenced the blood compatibility of the PU membrane. In addition to this, AS oil is reported to possess various chemical constituents and these constituents might also have a putative role in it. To corroborate this, FTIR analysis indicated the increase in $\mathrm{CH}$ peak intensity favouring the enhanced hydrophobicity resulting in improved blood compatibility [41,42].

\section{Conclusion}

In this study, the PU incorporated with AS oil was successfully electrospun using the electrospinning technique. The morphology analysis showed that the addition of AS oil in the PU membrane resulted in reduced fiber diameter compared to control. The presence of AS oil in PU membrane was confirmed through hydrogen bond formation. Further, the PU blended with AS oil showed hydrophobic and increase surface roughness than the pristine PU. Moreover, the blood compatibility assessments revealed that the developed biocomponent PU/AS oil membrane 
possess enhanced anticoagulant nature compared to control. Finally, this study suggests that the newly developed biocomponent PU/AS oil membrane with better physico-chemical characteristics and biological properties enabled them as a potential candidate for bone tissue engineering applications. It would be interesting to investigate the combined effect of $\mathrm{HA}$ in the biocomponent PU/AS membrane and this may throw further light on the feasibility of biocomponent PU/AS oil membrane in bone tissue engineering. Moreover, in vitro and in vivo testing on the fabricated biocomponent PU/AS oil membrane would make them suitability for bone tissue engineering.

\section{Acknowledgement}

This work was supported by the Ministry of Higher Education Malaysia with the Grant no. Q.J130000.2545.17H00 and Q.J130000.2545.20H00.

\section{Conflict of interest}

The authors declare that they have no conflict of interest.

\section{References}

1) Amini AR, Laurencin CT, Nukavarapu SP. Bone tissue engineering: recent advances and challenges. Critical Reviews ${ }^{\mathrm{TM}}$ in Biomedical Engineering. 2012;40(5).

2) Emam HE, Abdellatif FH, Abdelhameed RM. Cationization of celluloisc fibers in respect of liquid fuel purification. Journal of Cleaner Production. 2018 Mar 20;178:457-67.

3) Emam HE, Abdelhamid HN, Abdelhameed RM. Self-cleaned photoluminescent viscose fabric incorporated lanthanide-organic framework (Ln-MOF). Dyes and Pigments. 2018 Dec 1;159:491-8.

4) Emam HE, Abdelhameed RM. Anti-UV radiation textiles designed by embracing with nano-MIL (Ti, In)-metal organic framework. ACS applied materials \& interfaces. 2017 Aug 14;9(33):28034-45.

5) Emam HE, Abdelhameed RM. In-situ modification of natural fabrics by Cu-BTC MOF for effective release of insect repellent (N, N-diethyl-3-methylbenzamide). Journal of Porous Materials. 2017 Oct 1;24(5):1175-85.

6) Jaganathan SK, Mani MP, Palaniappan SK, Rathanasamy R. Fabrication and characterisation of nanofibrous polyurethane scaffold incorporated with corn and neem oil using single stage electrospinning technique for bone tissue engineering applications. Journal of Polymer Research. 2018 Jul 1;25(7):146.

7) Jaganathan SK, Mani MP, Nageswaran G, Krishnasamy NP, Ayyar M. Single stage electrospun multicomponent scaffold for bone tissue engineering application. Polymer Testing. 2018 Sep 1;70:244-54.

8) Emam HE. Generic strategies for functionalization of cellulosic textiles with metal salts. Cellulose. 2018:1-7. 
9) Emam HE. Antimicrobial cellulosic textiles based on organic compounds. 3 Biotech. 2019 Jan 1;9(1):29.

10) Ahmed HB, El-Hawary NS, Emam HE. Self-assembled AuNPs for ingrain pigmentation of silk fabrics with antibacterial potency. International journal of biological macromolecules. 2017 Dec 1;105:720-9.

11) Emam HE, Darwesh OM, Abdelhameed RM. In-growth metal organic framework/synthetic hybrids as antimicrobial fabrics and its toxicity. Colloids and Surfaces B: Biointerfaces. 2018 May 1;165:219-28.

12) Emam HE, El-Rafie MH, Ahmed HB, Zahran MK. Room temperature synthesis of metallic nanosilver using acacia to impart durable biocidal effect on cotton fabrics. Fibers and Polymers. 2015 Aug 1;16(8):1676-87.

13) Ahmed HB, Emam HE, Mashaly HM, Rehan M. Nanosilver leverage on reactive dyeing of cellulose fibers: color shading, color fastness and biocidal potentials. Carbohydrate polymers. 2018 Apr 15;186:310-20.

14) Walther A, Hoyer B, Springer A, Mrozik B, Hanke T, Cherif C, Pompe W, Gelinsky M. Novel textile scaffolds generated by flock technology for tissue engineering of bone and cartilage. Mater 2012;5(3):540-57

15) Huang ZM, Zhang YZ, Kotaki M, Ramakrishna S. A review on polymer nanofibers by electrospinning and their applications in nanocomposites. Compos Sci Technol 2003;63(15):2223-53.

16) O'brien FJ. Biomaterials \& scaffolds for tissue engineering. Mater Today 2011; 14(3): 8895.

17) Dhandayuthapani B, Yoshida Y, Maekawa T, Kumar DS. Polymeric scaffolds in tissue engineering application: a review. Int J Polym Sci 2011; 2011:1-19.

18) Sheikh FA, Barakat NA, Kanjwal MA, Chaudhari AA, Jung IH, Lee JH, Kim HY. Electrospun antimicrobial polyurethane nanofibers containing silver nanoparticles for biotechnological applications. Macromol Res 2009;17(9):688-96.

19) Lamba NMK, Woodhouse KA, Cooper SL. Polyurethanes in Biomedical Applications. Florida: CRC Press 1998.

20) Ma ZW, Hong Y, Nelson DM, Pichamuthu JE, Leeson CE, Wagner WR. Biodegradable polyurethane ureas with variable polyester or polycarbonate soft segments: Effects of crystallinity, molecular weight, and composition on mechanical properties. Biomacromol 2011;12:3265-3274.

21) Unnithan AR, Tirupathi PB, Gnanasekaran G, Seenivasan K, Barakat Nasser AM, Jung YS, Shanmugam A, Kim HY. Emu oil-based electrospun nanofibrous scaffolds for wound skin tissue engineering, Colloids Surf A: Physicochem Eng Asp 2012; 415: 454-460

22) Chen PH, Liao HC, Hsu SH, Chen RS, Wu MC, Yang YF, Wu CC, Chen MH, Su WF. A novel polyurethane/cellulose fibrous scaffold for cardiac tissue engineering. RSC Adv. 2015;5(9):6932-9. 
23) Tetteh G, Khan AS, Delaine-Smith RM, Reilly GC, Rehman IU. Electrospun polyurethane/hydroxyapatite bioactive Scaffolds for bone tissue engineering: The role of solvent and hydroxyapatite particles. J Mech Behav Biomed Mater. 201439:95-110.

24) Chao CY, Mani MP, Jaganathan SK. Engineering electrospun multicomponent polyurethane scaffolding platform comprising grapeseed oil and honey/propolis for bone tissue regeneration. Plos one. 2018;13(10):e0205699.

25) Remya NS, Syama S, Sabareeswaran A, Mohanan PV. 2017. Investigation of chronic toxicity of hydroxyapatite nanoparticles administered orally for one year in wistar rats. Mater Sci Eng: C. 2017;76:518-27.

26) Shahbazi MA, Herranz B, Santos HA. Nanostructured porous Si-based nanoparticles for targeted drug delivery. Biomatter. 2012;2(4):296-312.

27) Radhakrishnan S, Bhavan PS, Seenivasan C, Shanthi R, Poongodi R. Influence of medicinal herbs (Alteranthera sessilis, Eclipta alba and Cissus quadrangularis) on growth and biochemical parameters of the freshwater prawn Macrobrachium rosenbergii. Aqua Inter. 2014;22(2):551-72.

28) Naples ML. Weeds of rain fed lowland rice fields of Laos and Cambodia. Unpublished M.Sc thesis. University of Leiden, The Netherlands. 2005.

29) Song-Chow L, Yun-Ho L, Shyh-Jong S, Chung-Ching L. Hepatoprotective effects of Taiwan folk medicine: Alternanthera sessilis on liver damage induced. Phytother Res. 2006; 8:391-398

30) Abulude FO. Nutritional evaluation of aquatic weeds in Nigeria. E J Environ Agricult Food Chem. 2005;4:835-840

31) Mohammadi A, Barikani M, Barmar M. Synthesis and investigation of thermal and mechanical properties of in situ prepared biocompatible Fe $3 \mathrm{O}$ 4/polyurethane elastomer nanocomposites. Polym Bulletin. 2015;72(2):219-34.

32) Tijing LD, Ruelo MTG, Amarjargal A, Pant HR, Park C-H, Kim DW, Kim CS. Antibacterial and superhydrophilic electrospun polyurethane nanocomposite fibers containing tourmaline nanoparticles. Chem Eng J 2012; 197: 41-48.

33) Cui W, Li X, Zhou S, Weng J. Degradation patterns andsurface wettability of electrospun fibrous mats. Polym Degrad Stab. 2008;93(3):731-738.

34) Ceylan M. Superhydrophobic behavior of electrospun nanofibers with variable additives. 2009: Available at: http://hdl.handle.net/10057/2535

35) Džunuzović JV, Pergal MV, Poręba R, Vodnik VV, Simonović BR, Špírková M, Jovanović $\mathrm{S}$. Analysis of dynamic mechanical, thermal and surface properties of poly (urethane-estersiloxane) networks based on hyperbranched polyester. Journal of Non-Crystalline Solids. 2012 Dec 1;358(23):3161-9.

36) Król P. Linear polyurethanes: synthesis methods, chemical structures, properties and applications. CRC Press; 2008 Mar 27. 